\title{
Finding Zeroes of Maps: Homotopy Methods That Are Constructive With Probability One*
}

\author{
By Shui-Nee Chow, John Mallet-Paret and James A. Yorke
}

\begin{abstract}
We illustrate that most existence theorems using degree theory are in principle relatively constructive. The first one presented here is the Brouwer Fixed Point Theorem. Our method is "constructive with probability one" and can be implemented by computer. Other existence theorems are also proved by the same method. The approach is based on a transversality theorem.
\end{abstract}

1. Introduction. Degree theory has become a major tool in analysis. It is generally used to prove the existence of solutions for a wide variety of problems. Other methods of proof, such as those based on the Implicit Function Theorem, generally require detailed information about the functions involved, or the results are local. In degree theory, proofs are topological in nature and require relatively little prior information about the location of the supposed solutions and little need be assumed about the detailed behavior of the mappings involved. By comparison, methods based on the Implicit Function Theorem are used to guarantee existence of solutions near other known solutions. Another method of analysis is the use of contracting maps which requires substantial information on the point-to-point behavior of the maps involved. In return for the stronger assumptions required in order to employ these analytical approaches, we expect the proofs to be constructive. In this paper we illustrate that most degree theory proofs involving smooth functions are in principle rather constructive. Our numerical experience has shown us that apparently nonconstructive degree theory proofs can be readily turned into fairly constructive proofs involving an algorithm that can be implemented on computers.

The methods we describe are constructive (or implementable) only in a somewhat restrictive sense, a sense which is useful in practice. For each given problem there is associated a set $A \subset R^{m}$ which has nonempty interior. We say a procedure is "constructive with probability one" if for almost every $a \in A$ with respect to $m$ dimensional Lebesgue measure there is a given point $p_{a}$ and a path $\Gamma_{a}\left(\Gamma_{a}\right.$ is the trajectory leading from $p_{a}$ for a known ordinary differential equation in some Euclidean space) and this path from $p_{a}$ "leads" to point $s_{a}$, which is a solution of the given problem, or perhaps the projection of this point $s_{a}$ into some lower-dimensional space is a solu-

Received November 30, 1976; revised August 25, 1977.

AMS (MOS) subject classifications (1970). Primary 90B99. Secondary 65H10, 65L10, 58C99, 47H10, 54H25, $55 \mathrm{C} 20$.

Key words and phrases. Brouwer Fixed Point Theorem, constructive proof, Transversality theorem, degree theory, vector fields on spheres.

* This research was partially supported by National Science Foundation grants NSF 71-1577 (Chow), MCS 76-0724 (Mallet-Paret), and MCS 76-24432 (Yorke) and also under grand ARO-AAG29-76-G-0294 (Mallet-Paret). 
tion. The curve $\Gamma_{a}$ lies in a compact set but may in some case have infinite length. Then we require that every point in the positive set of $\Gamma_{a}$ (or the projection of these points) must be a solution to the given problem.

To describe the limitations of the computer implementability, we describe now a particularly simple problem: solving for the roots of a polynomial $p_{0}(z)=\Sigma b_{i} z^{i}=$ 0 where $b_{i} \in \mathbf{C}$ for $i=0, \ldots, k, b_{k}=1$. One proof of existence is based on the topological degree of the map $p_{0}: \mathbf{C} \rightarrow \mathbf{C}$. For $a \in \mathbf{C}^{k}, a=\left(a_{1}, \ldots, a_{k}\right)$ let

$$
p(a, z)=\prod_{i=1}^{k}\left(z-a_{i}\right) .
$$

For almost any $a \in \mathbf{C}^{n}$, the set of solutions of

$$
(1-t) p(a, z)+t p_{0}(z)=0, \quad 0 \leqslant t \leqslant 1,
$$

is $k$ paths in $(t, z)$ space connecting $\left(0, a_{i}\right)$ to $\left(1, z_{i}\right)$ where $z_{i}$ is a solution, a root of $p_{0}$. Each of these curves is the trajectory of an ordinary differential equation and may be closely followed numerically. Hence, we say the method is constructive with probability one. Of course, it is possible that $k$ is so large that it even exceeds the storage capacity of the computer. Also, the computer can represent only finitely many points in $\mathbf{C}$, and this may cause difficulties; and errors arise inevitably in following trajectories, numerically. The main problem in implementing degree theoretic existence theorems is that initially there is no idea where the solution might be. Once a good approximation is obtained a number of techniques may then become available, so from this point of view it is sufficient to obtain a good numerical approximation to the trajectory. In practice these methods yield $\epsilon$-approximate solutions so that one might expect to find a point $z_{\epsilon}$ such that $\left|p_{0}\left(z_{\epsilon}\right)\right|<\epsilon$. In practice $\epsilon$ can be made quite small $\left(10^{-10}\right.$ or less). Of course, a pathological example can be constructed for any $\epsilon$ so that there will exist such a $z_{\epsilon}$ which is not close to an actual root. However, if the objective is to construct pathological examples, much more absurd situations are possible. Even if it is known that all solutions of $p_{0}(z)=0$ lie in the unit disc, any algorithm must contend with the difficulty that the computer represents only a finite number of points in the disc and $p_{0}(z)$ might be chosen so pathologically that $p_{0}(z) \geqslant 1$ for all the computers' points. We explicitly ignore these difficulties in our use of the phrase "constructive with probability one".

The history of algorithms for implementing degree-theoretic arguments starts with the Brouwer Fixed Point Theorem.

Let $R^{n}$ be the real $n$-dimensional space with norm $|\cdot|, B^{n}=\left\{x \in R^{n}:|x|<1\right\}$ and $\bar{B}^{n}=\left\{x \in R^{n}:|x| \leqslant 1\right\}$ and $S^{n-1}=\left\{x \in R^{n}:|x|=1\right\}$. For any continuous mapping $f: \bar{B}^{n} \rightarrow \bar{B}^{n}$, the Brouwer Fixed Point Theorem guarantees the existence of a fixed point $x_{0} \in \bar{B}^{n}$. The associated numerical problem is to give a constructive proof of the theorem, or give an algorithm for finding a fixed point.

A numerical method which can be implemented by computer was discovered 
by Scarf [1]. His method is based on Sperner's lemma. The ball $\bar{B}^{n}$ (assumed to be a simplex) is simplicially decomposed and the method produces a path along the edges in the simplicial decomposition. The path will of course lead to a fixed point of a piecewise linear approximation of $f$. This method was extended and simplified in a number of ways. See, for example, [2]-[6], [17] .

Recently, nonsimplicial approaches were found by Kellogg, Li and Yorke [7], [8]. Smale [9] recently discovered similar ideas for finding zeroes of maps and applied them to economics. Hirsch and Smale [10] extended the approach to maps of $R^{n}$ into $R^{n}$. These approaches, which may be called "continuation methods" or "Davidenko's method", are based on the following ideas. Pontrjagin [11] studied homotopy properties of maps of spheres to spheres of one dimension lower. He used Sard's Theorem to study inverse images of points. In [12], Hirsch then proved the Brouwer Fixed Point Theorem as follows. He assumes no solutions exist and employs the standard retraction $g: \bar{B}^{n} \rightarrow \partial \bar{B}^{n}=S^{n-1}$, where $g(x)$ is the point where $S^{n}$ meets the ray from $f(x)$ through $x$. Assuming $f$ is smooth and has no fixed points, he uses Sard's Theorem to investigate the set $g^{-1}(y)$ for a regular value $y \in \partial \bar{B}^{n}$. He eliminates all possibilities for what it can be and, thereby, obtains a contradiction. Hence, there must be a fixed point of $f$. In [7] the argument was modified slightly to make it constructive with probability one; the mapping $g$ was considered to be defined everywhere in $\bar{B}^{n}$ except at the fixed points of $f$. Now for any regular value $y \in S^{n-1}$ we have $g^{-1}(y)$ is the union of a curve leading from $y$ to a subset of the fixed points of $f$, plus disjoint closed paths. An algorithm was then given for following the curve from $y$ to the fixed points. In [8] it is reported the Brouwer Fixed Point was found for some 20 dimensional problems in an average time of 3.3 seconds using the UNIVAC 1108 . The method for following the curve is made rigorous in [13]. Further numerical experiments have been carried out by Watson [14] in response to a preprint of this paper.

In this paper, we give a more general approach based on a transversality theorem which we call "the Parametrized Sard Theorem". This approach will give solutions to problems in which the topological scheme is more complicated. In particular, we investigate constructive methods for the following problems:

1. Finding the Brouwer fixed point;

2. Finding zeroes of maps of $R^{n}$ into $R^{n}$ under various hypotheses;

3. Finding solutions of two point boundary problems for second order ordinary differential equations in $R^{n}$;

4. Finding zeroes of a vector field on $S^{n}$, where $n$ is even.

The basic idea in our approach is simple and can be described briefly as follows. Consider the mapping $f$ (defined on $\bar{B}^{n}$ or Euclidean space or $S^{n}$ ) whose zeroes (or fixed points) are desired and assume we are given a "trivial" mapping $g_{a}$ whose zeroes are known a priori, depending on parameter $a$. Define the homotopy

$$
\phi_{a}(\lambda, x)=(1-\lambda) g_{a}(x)+\lambda f(x), \quad 0 \leqslant \lambda \leqslant 1 .
$$

We use the general "Sard" theorems to obtain a guarantee that for almost every $a$, 
$\phi_{a}^{-1}(0)$ contains a smooth curve $\Gamma_{a}$ (see Figure 1), a curve which will lead from a zero of $g_{a}$ to a zero of $f$.

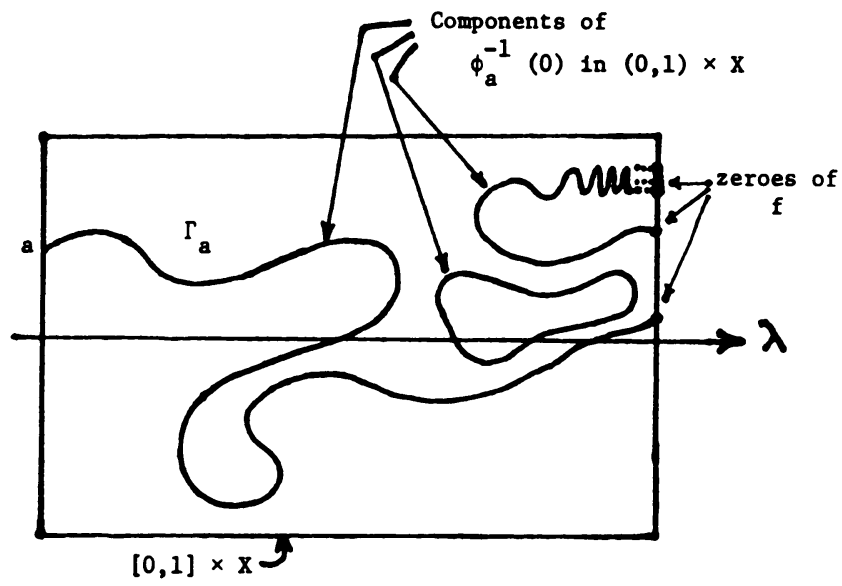

Figure 1

The continuation method for finding a zero of a map $f: X \rightarrow R^{n}$, a possible configuration. In this figure a trivial map at $\lambda=0$ has $a$ as its unique zero. $\Gamma_{a} \subset(0,1) \times X$ is the component of $\phi_{a}^{-1}(0)$ which has $(0, a)$ on its boundary. $\Gamma_{a}$ leads from $a$ at $\lambda=0$ to a zero of $f$ at $\lambda=1$. Numerical algorithms are available for following $\Gamma_{a}$. As the curve is followed from $(0, a), \lambda$ need not increase monotonically but this causes no numerical difficulties.

Solutions have often been shown to exist by constructing a homotopy from a trivial map to the one of interest. This is a basic technique for example in LeraySchauder degree theory. Homotopy methods are used in [15] for constructively finding fixed points for simplicial maps. Our contribution here is the idea that one should start with a large class of trivial maps. Next choose one at random. Next homotop it to the nontrivial map. Then with probability one, a curve starting from the fixed point of the trivial map will lead us by a smooth path to a fixed point of the nontrivial map.

Homotopy methods for finding zeroes or fixed points are known variously as the "Continuous Newton's Method" or the continuation method or Davidenko's method. See, for example, [16].

The history of this paper is as follows. The basic ideas of this paper, which use the Parametrized Sard Theorem, were presented by Yorke at the 1976 NSF regional conference in Boulder, Colorado. He also proposed the problem of finding a constructive proof of the existence of zeroes of vector fields on even-dimensional spheres. Chow and Mallet-Paret, who attended this meeting, found how to solve this sphere problem using the Transversality Theorem.

Acknowledgements. We would like to thank M. Hirsch, H. Scarf, and S. Smale for enlightening discussions.

2. Generalizations of Sard's Theorem. Let $U \subset R^{n}$ be open and $f: R^{n} \rightarrow R^{p}$ be smooth. We say $y \in R^{p}$ is a regular value for $f$ if 


$$
\text { Range } D f(x)=R^{p} \quad \text { for all } x \in f^{-1}(y)
$$

where $D f(x)$ denotes the $n \times p$ matrix of partial derivatives of $f(x)$. Sard's Theorem says that if $f$ is smooth (or $C^{r}, r>\max (0, n-p)$ ), then almost every $y \in R^{p}$ is regular. See $[18$, p. 7$]$. We now state the more general "Parametrized Sard's Theorem".

THEOREM 2.1. Let $V \subset R^{q}, U \subset R^{m}$ be open and let $\phi: V \times U \rightarrow R^{p}$ be $C^{r}, r>\max \{0, m-p\}$. If $0 \in R^{p}$ is a regular value of $\phi$, then for almost every $a \in V, 0$ is a regular value of $\phi_{a}(\cdot)=\phi(a, \cdot)$.

We will usually be interested in the case $q=p=n, m=n+1$. Our assumption will guarantee 0 is a regular value of $\phi$; since Range $D_{(a, x)} \phi=$ Range $D_{a} \phi+$ Range $D_{x} \phi$, it will be sufficient to guarantee Range $D_{a} \phi=R^{p}$. Here $D_{a} \phi$ denotes the matrix of partial derivatives of $\phi$ with respect to $a$, so $D_{a} \phi(a, x)$ is a $q \times p$ matrix.

Proof. The proof of Theorem 2.1 may be found in [17]. For the convenience of the reader, we include the following outline of the proof. By the Implicit Function Theorem, $M=\phi^{-1}(0)$ is a smooth manifold in $R^{q+m}$. Define the projection

$$
\pi: M \rightarrow R^{q} \text { by } \pi(a, x)=a .
$$

By Sard's Theorem, almost every $a \in R^{q}$ is a regular value of $\pi$. But the regularity of $a$ for $\pi$ is equivalent to the condition: if $(a, x) \in M$, then for all $b \in R^{q}$ there exists $y \in R^{m}$ such that $(b, y)$ is tangent to $M$ at $(a, x)$, that is

$$
D \phi(a, x) \cdot(b, y)=0
$$

that is,

$$
D_{a} \phi(a, x) \cdot b+D_{x} \phi(a, x) \cdot y=0 \text {. }
$$

By the regularity of $0 \in R^{p}$ and the above equation, we have

$$
\text { Range } D_{x} \phi(a, x)=\text { Range } D \phi(a, x)=R^{p} \text {. }
$$

A slightly stronger version will be needed in Section 6; it is easy to see that Theorem 2.1 is still true if $\phi$ is a smooth mapping between smooth finite-dimensional manifolds. In fact, the following is true, [17] .

Theorem 2.2 (Transversality Theorem). Let $Q, N$ and $P$ be smooth manifolds of dimensions $q, m$ and $\tilde{p}$, respectively. Let $W \subset P$ be a submanifold of codimension $p$. (That is, the dimension of $P=p+$ dimension of $W$.) Consider a smooth mapping

$$
\phi: Q \times N \rightarrow P .
$$

If $\phi$ is transversal to $W$, then for almost every $a \in Q, \phi_{a}(\cdot)=\phi(a, \cdot): N \rightarrow P$ is transversal to $W$.

(Recall that a smooth mapping $h: N \rightarrow P$ is transversal to $W$ if $\{$ Range $D h(x)\}+\left\{T_{y} W\right\}=T_{y} P \quad$ whenever $y=h(x) \in W$, 
where $T_{y} W$ and $T_{y} P$ denote the tangent spaces of $W$ and $P$ at $y$, respectively.)

Given the hypotheses of Theorems 2.1 (or 2.2 , respectively) we may draw the following standard conclusion from the Implicit Function Theorem. See [18, Appendix] .

COROLlARY 2.3. If $m=p+1$, then each component of $\phi_{a}^{-1}(0)$ (or $\phi_{a}^{-1}(W)$, respectively) is a smooth curve for almost every $a$ in $V$ (or in $Q$ ) with respect to $q$ dimensional Lebesgue measure.

Hence, we may say that if we choose a point $a$ at random from $V$ (or $Q$ ), the probability is one that each component of $\phi_{a}^{-1}(0)$ (or $\phi_{a}^{-1}(W)$ ) is a smooth curve. In practice, we will know one point of $\phi_{a}^{-1}(0)$ and will write $\Gamma_{a}$ for the component that that point is in. More generally, we will be able to distinguish a particular component of $\phi_{a}^{-1}(0)$, and it will be denoted $\Gamma_{a}$. Algorithms have been presented for following this curve to its other end, so the methods based on results in this section are constructive with probability one. See Section 7 .

Degree theory results are based on homotopy ideas. We now investigate in detail the homotopy mapping $\phi$ we will use for the Brouwer Fixed Point Theorem. For other problems we change $\phi$ slightly if we are investigating zeroes of a map $f$ instead of fixed points, and the domain and range of $\phi$ will vary from problem to problem. In all cases we investigate $\phi_{a}^{-1}(0)$.

Let $f: \bar{B}^{n} \rightarrow \bar{B}^{n}$ be smooth, and define

$$
g(a, x)=x-a, \quad a, x \in R^{n}, \quad \text { and } \quad \phi(a, \lambda, x)=(1-\lambda) g(a, x)+\lambda(x-f(x)) .
$$

In seeking fixed points of $f$, we examine the zeroes of $g$ and $\phi$. Let $\Gamma_{a}$ be the component of $\phi_{a}^{-1}(0) \cap(0,1) \times \bar{B}^{n}$ whose closure contains $(0, a)$.

THEOREM 2.4. Consider the mapping

$$
\phi: B^{n} \times(0,1) \times B^{n} \rightarrow R^{n}
$$

defined as above. We have

(a) $0 \in R^{n}$ is a regular value of $\phi$,

(b) for almost every $a \in B^{n}, \Gamma_{a}$ is a smooth curve in $(0,1) \times B^{n}$ joining $(0, a)$ to a fixed point of $f$ (or to a set of fixed points) at $\lambda=1$.

Remark. In practice for most $f$ the curve $\Gamma_{a}$ forms a smooth arc leading to a fixed point of $f$. However, possibilities remain that $\Gamma_{a}$ will not actually meet the fixed point but will converge to a set of fixed points of $f$ as shown in Figure 1. Hence, $\phi_{a}^{-1}(0)$ is smooth when restricted to $(0,1) \times B^{n}$ or $[0,1) \times B^{n}$ but not necessarily in $[0,1] \times \bar{B}^{n}$.

Proof. Let $(\bar{a}, \bar{\lambda}, \bar{x}) \in B^{n} \times(0,1) \times B^{n}$ and $\phi(\bar{a}, \bar{\lambda}, \bar{x})=0$. Then $D_{a} \phi(\bar{a}, \bar{\lambda}, \bar{x})=-(1-\lambda) I, I=$ identity matrix, and for $\lambda \neq 1$,

$$
\text { Range } D \phi(\bar{a}, \bar{\lambda}, \bar{x}) \supset \text { Range } D_{a} \phi(\bar{a}, \bar{\lambda}, \bar{x})=R^{n} \text {. }
$$

This proves (a). By Theorem 2.1, for almost every $a \in B^{n}, 0$ is a regular value of $\phi_{a}$. By Corollary $2.3, \phi_{a}^{-1}(0)$ is a smooth curve in $(0,1) \times B_{n}$. Hence, each component is a smooth curve and is either diffeomorphic to a circle or an open interval. 
Consider the map $\phi_{a}$ with domain $(-\infty, 1) \times \bar{B}^{n}$. Since $\phi_{a}(0, a)=0$ and $D_{x} \phi_{a}(0, x)=I$, we may solve for $|\lambda|<<1$ in a neighborhood of $(0, a)$ by the Implicit Function Theorem, and write

$$
\phi_{a}(\lambda, x(\lambda))=0, \quad \text { where } x(0)=a .
$$

This implies that $\Gamma_{a} \subset(0,1) \times \bar{B}^{n}$ is not diffeomorphic to a circle. It remains to be shown that $\Gamma_{a}$ has no limit points on the lateral surface $(0,1) \times S^{n-1}$. Such a limit point $(\lambda, x)$ would satisfy

$$
\phi_{a}(\lambda, x)=0, \quad \text { where } 0<\lambda<1,
$$

then $x=(1-\lambda) a+\lambda f(x)$. Hence, $x$ is on the line segment between $a$ and $f(x)$.

Since $a \in B^{n}, f(x) \in \bar{B}^{n}$, we have $x \in B^{n}$. Hence, $\Gamma_{a}$ has no limit points on $(0,1) \times S^{n-1}$. Of course, any limit point of $\Gamma_{a}$ is in $\phi_{a}^{-1}(0)$ so the only limit point of $\Gamma_{a}$ in $\{0\} \times B^{n}$ is $(0, a)$. Furthermore, we have seen that $\Gamma_{a}$ is homeomorphic to an open interval and $(0, a)$ lies at one end. By compactness of $[0,1] \times B^{n}$ there must be at least one additional limit point of $\Gamma_{a}$ and all such limit points must lie in $\{1\} \times B^{n}$. If $\left(1, x_{1}\right)$ is such a point, then $x_{1}$ is a fixed point of $f$.

Notice that if $I-D f(x)$ is nonsingular for every fixed point $x$ of $f$, then $\bar{\Gamma}_{a}$ is a smooth curve in $[0,1] \times \bar{B}^{n}$ and so has finite arc length.

3. The Brouwer Fixed Point Theorem. We may immediately deduce the Brouwer Fixed Point Theorem from Theorem 2.4 when $f$ is smooth. Suppose now $f$ is merely continuous. Approximating $f$ by smooth mappings $f_{k}, k=1,2, \ldots$, we obtain a sequence of smooth curves $\Gamma_{a}^{k}$. By taking the linit of these curves $\bigcap_{n=1}^{\infty} \bigcup_{k \geqslant n} \Gamma_{a}^{k}$

$$
\phi_{a}^{-1}(0) \cap\left(\{1\} \times \bar{B}^{n}\right) \neq \varnothing .
$$

We now have that for any smooth $f: \bar{B}^{n} \rightarrow \bar{B}^{n}$ we may find a fixed point by following a curve $\Gamma_{a}$ of fixed points we get by starting with the trivial map $x-a=0$, for almost any $a$.

Mas-Colell [19] has applied the Thom transversality theorem to obtain a related result. He says that for $f$ in an open and dense subset of the twice differentiable functions, we may find the fixed point by starting with the trivial map $x=0$. That is, the set of solutions of $\lambda f(x)-x=0$ will be the union of a set of smooth curves for "most choices of $f$." See also [20].

4. Maps of $R^{n}$ into $R^{n}$. There are many different variants of Brouwer's Fixed Point Theorem. Some are used in the existence proof for nonlinear partial differential equations. For example, in monotone operator theory the following version of Brouwer's Fixed Point Theorem is used in connection with Galerkin's approximation (see [21, pp. 53-56 and 171-173]).

THEOREM 4.1. Let $f: R^{n} \rightarrow R^{n}$ be smooth. Suppose that

$$
f(x) \cdot x \geqslant 0 \text { when }|x|=1 .
$$

Then there exists $|x| \leqslant 1$ such that $f(x)=0$. 
Proof. The standard proof is by contradiction [21, p. 53]. We present here a constructive proof similar to the previous one. Consider the mapping

$$
\phi(a, \lambda, x)=(1-\lambda)(x-a)+\lambda f(x), \quad|a|<1,0<\lambda<1 .
$$

By similar arguments as in the proof of Theorem 2.4, we obtain for almost every $a \in B^{n}$ a smooth curve $\Gamma_{a} \subset \phi_{a}^{-1}(0)$ of zeros of $\phi_{a}$. To see that $\Gamma_{a}$ continues to $\lambda=1$, we must show $\Gamma_{a}$ cannot contain any points $(\lambda, x)$ with $0<\lambda<1$ and $x \in$ bnd $B^{n}$. Suppose otherwise, that there is $\lambda \in(0,1)$ and $x$ with norm 1 such that

$$
(1-\lambda)(x-a)+\lambda f(x)=0 .
$$

Using (4.1), we get $(x-a) \cdot x \leqslant 0$ since $(1-\lambda)>0$. Hence $1 \leqslant a \cdot x$ which is impossible since $|a|<1$, which is a contradiction. Since $\Gamma_{a}$ has one endpoint at $(0, a)$, its other endpoint must lie at $(1, \tilde{x})$ where $\tilde{x}$ is a zero of $f$.

Hirsch and Smale [10] have used continuation to give a constructive proof for several similar results and also for the following theorem.

THEOREM 4.2. Let $f: R^{n} \rightarrow R^{n}$ be smooth. Suppose that $|f(x)| \rightarrow \infty$ as $|x| \rightarrow \infty$. Suppose for some $\beta>0$, we have det $D f(x) \geqslant 0$ when $|f(x)|>\beta$ and suppose we are given a sequence $\left\{x_{i}\right\}$ with $\left|x_{i}\right| \rightarrow \infty$ as $i \rightarrow \infty$ such that $\operatorname{det} D f\left(x_{i}\right)>0$. Assume $n>1$. Then $f$ has a zero.

The continuation approach they use does not explicitly mention homotopies, but it is equivalent to the following approach. Let $f(x)-f(a)$ be the trivial map. (It is "trivial" because we immediately know $x=a$ is a zero.) Then let

$$
\phi(a, \lambda, x)=(1-\lambda)[f(x)-f(a)]+\lambda f(x)=f(x)-(1-\lambda) f(a) .
$$

Now let $V$ be an open subset of $R^{n}$ on which $|f(x)|>\beta$ and det $D f(x) \neq 0$. Hence, $D_{a} \phi$ has rank $n$ for $\lambda<1$ and so 0 is a regular value for $\phi_{a}$ for almost every $a \in V$. Using degree theory arguments which we cannot give here, Hirsch and Smale have shown in effect that the curve $\Gamma_{a}$ is bounded and leads to a zero of $f$ for almost every $a \in V$.

J. Alexander [22] has found an application of this result to the following problem. Given any complex $k \times k$ matrix $\left(m_{i j}\right)$ and the complex number $\lambda_{1}, \ldots, \lambda_{k}$, how may one change the diagonal entries $m_{11}, \ldots, m_{k k}$ so the altered matrix has $\lambda_{1}, \ldots, \lambda_{k}$ as its eigenvalues? For all matrices there are $k$ ! ways that the diagonal $\left\{m_{11}, \ldots, m_{k k}\right\}$ may be chosen (for almost any choice of $\left.\left\{\lambda_{1} \cdots \lambda_{k}\right\}\right)$. While Friedland had previously shown [23] that it is possible to choose the diagonal appropriately, Alexander [22] has shown how Theorem 4.2 is always applicable and so can be used constructively to find appropriate diagonals.

5. Solving a Two Point Boundary Value Problem. While we may think of the Brouwer Fixed Point Theorem as being a basic tool in problems of applied mathematics, surprisingly few mathematical applications outside mathematical economics have been found for the constructive methods first introduced by Scarf. We feel that by the simple modifications suggested in this paper, a richer class of problems becomes tractable. We present this section as an illustration. See also [4]. 
Let $\psi: R^{k} \times R^{k} \rightarrow R^{k}$ be smooth and consider the second order boundary value problem on $[-1,+1]$,

$$
\left.\begin{array}{l}
u^{\prime \prime}=\psi\left(u, u^{\prime}\right) \\
u(-1)=0=u(1)
\end{array}\right\} B V P .
$$

Theorem 5.1 (Scorza-Dragoni [24]). Assume $|\psi|$ is bounded. Then BVP has at least one solution.

Our objective here is to find a constructive continuation method for producing the solution. The simplest nonconstructive approach would be to consider the trivial equation $u^{\prime \prime}=0$ and homotop the 0 map to $\psi$, all the while using the boundary condition (5.2). The standard Leray Schauder (or Brouwer) degree argument would be to prove that there is a uniform bound for all solutions of all intermediate problems, that is for $u^{\prime \prime}=\lambda f\left(u, u^{\prime}\right)$ with (5.2). Such a method is used for more general boundary value problems by Lasota and Yorke [25]. This approach need not produce a smooth curve of solutions and so it is not "constructive" in our sense.

Continuation Proof. Consider the trivial problem

$$
u^{\prime \prime}=0, \quad u(-1)=a_{0}, \quad u(+1)=a_{1},
$$

where $a_{0}, a_{1} \in R^{k}$. We now homotop this problem to the problem $B V P$. For $\lambda \in[0,1]$ we consider

$$
\left.\begin{array}{l}
u^{\prime \prime}=\lambda \psi\left(u, u^{\prime}\right) \\
u(-1)=(1-\lambda) a_{0}, u(+1)=(1-\lambda) a_{1}
\end{array}\right\} B V P_{\lambda} .
$$

Let $w$ be $\left(v_{0}, v_{1}, \lambda\right) \in R^{2 k+1}$ and write $u(t, w)$ for the solution of (5.3) for which

$$
u(0, w)=v_{0} \quad \text { and } \quad u^{\prime}(0, w)=v_{1} .
$$

Let $a$ be $\left(a_{0}, a_{1}\right) \in R^{2 k}$ and define $\phi: R^{2 k} \times R^{2 k+1} \longrightarrow R^{2 k}$ by

$$
\phi(a, x)=\left(\lambda a_{0}-u(-1, w), \lambda a_{1}-u(1, w)\right) .
$$

Then $D_{a} \phi=\lambda$ where $I$ is the identity on $R^{2 k}$. Thus, Range $D_{a} \phi$ is all of $R^{2 k}$ for $\lambda<1$ and so the same must be true for Range $D \phi$. Therefore, we may apply Theorem 2.1.

Since $\psi$ is bounded, we may choose $\beta>0$ such that $|\psi| \leqslant \beta$ everywhere on $R^{2 k}$. Given $a_{0}, a_{1}$, let $\alpha=\max \left\{\left|a_{0}\right|,\left|a_{1}\right|\right\}$. It follows that if $u(t)$ is a solution of $B V P_{\lambda}$ for some $\lambda \leqslant 1$, then $|u(t)| \leqslant \alpha+\beta / 2$ for all $t \in[-1,+1]$. See [25] for more complicated estimates.

The argument now follows as before. The $\Gamma_{a}$ leads to a zero of $\phi_{a}$ with $\lambda=1$ for almost all $a$; of course this "solution" gives us $w=\left(v_{0}, v_{1}, 1\right)$ and $u(\cdot, w)$ is a solution of the original problem $B V P$.

In [24], [25] continuity of $\psi$ is assumed instead of smoothness and the initial value problems need not have unique solutions. Hence, the degree arguments are carried out in a function space. 
6. Vector Fields on Spheres. A basic result in bifurcation theory concerning eigenvalues with odd multiplicities is equivalent to saying any smooth vector field on an even-dimensional sphere must have a zero; (see Krasnoselskii [26]). Computational techniques for finding such zeroes are desirable.

Let $T S^{n}$ be the tangent bundle of $S^{n}$. Given a smooth vector field $\tau: S^{n} \rightarrow T S^{n}$, write

$$
\tau(x)=(x, f(x)),
$$

where $x \in S^{n}, x \cdot f(x)=0$ and $x, f(x) \in R^{n+1}$. For any $a \in S^{n}$ define the "trivial" vector field

$$
\sigma(x)=(x, g(a, x)), \quad g(a, x)=a-(a \cdot x) x
$$

( $\sigma$ is a vector field whose trajectories lead from the south pole " $-a$ " to the north pole " $a$ "). Let $\phi$ be the homotopy between $\sigma$ and $\tau$ defined by

$$
\phi(a, \lambda, x)=(x,(1-\lambda) g(a, x)+\lambda f(x)) .
$$

THEOREM 6.1. For the mapping

$$
\phi: S^{n} \times(0,1) \times S^{n} \rightarrow T S^{n}
$$

defined by (6.1) we have

(a) $\phi$ is transversal to $W=\left\{(x, y) \in T S^{n}: y=0\right\}$;

(b) if $n$ is even, then for almost every $a, \Gamma_{a}=\phi_{a}^{-1}(W)$ contains a curve in $(0,1) \times S^{n}$ such that $\Gamma_{a}$ leads from a to a zero of the vector field $\tau$ defined by

Proof. Suppose that $\phi(\bar{a}, \bar{\lambda}, \bar{x}) \in W$. Hence,

$$
(1-\bar{\lambda}) g(\bar{a}, \bar{x})+\bar{\lambda} f(\bar{x})=0 .
$$

Since $\bar{\lambda} \neq 0,1, g(\bar{a}, \bar{x})$ and $f(\bar{x})$ are proportional. Thus,

$$
\begin{aligned}
& \text { Range } D_{a} \phi(\bar{a}, \bar{\lambda}, \bar{x})=\{b-(b \cdot \bar{x}) \bar{x}: \bar{a} \cdot b=0\}, \\
& \text { Range } D_{\lambda} \phi(\bar{a}, \bar{\lambda}, \bar{x})=\{(\bar{a}-(\bar{a} \cdot \bar{x}) \bar{x}) \mu: \mu \in R\}
\end{aligned}
$$

Moreover,

$$
T_{\phi(\bar{a}, \bar{\lambda}, \bar{x})} W=\{y: y \cdot \bar{x}=0\}
$$

It is clear that

$$
\text { Range } D_{a} \phi(a, \lambda, x)+\text { Range } D_{\lambda} \phi(a, \lambda, x)=T_{\phi(a, \lambda, x)} W .
$$

This proves (a). Next, by Theorem 2.2 for almost every $a \in S^{n}, \phi_{a}$ is transversal to $W$. Since codim $W=\operatorname{codim} \phi_{a}^{-1}(W)=n$. Thus, $\phi_{a}^{-1}(W)$ is diffeomorphic to either a circle or an open interval. Just as in Theorem 2.4 it follows from the Implicit Function Theorem that $\Gamma_{a}=\phi_{a}^{-1}(W)$ is diffeomorphic to an open interval. 
Since the manifold $(0,1) \times S^{n}$ has no boundary, $\Gamma_{a}$ must have limit points on $\{0\} \times S^{n}$ or $\{1\} \times S^{n}$. Suppose that $\Gamma_{a}$ has no limit points on $\{1\} \times S^{n}$. Then, $\Gamma_{a}$ has two limit points, namely, $(0, a)$ and $(0,-a)$. Let $\Omega \subset(0,1) \times S^{n}$ be an open neighborhood of $\Gamma_{a}$ such that the boundary $\partial \Omega$ of $\Omega$ contains no points of $\{1\} \times S^{n}$. Let $\Omega(\lambda)=$ $\{x:(\lambda, x) \in \Omega\}$. Since $n$ is even, the indices of the vector field $\phi_{a}(0, \cdot)$ at $a$ or $-a$ are both equal to 1 . Thus,

$$
\operatorname{deg}\left(\phi_{a}(\lambda, \cdot), \Omega(\lambda)\right)=2 \text { for } 0<\lambda<<1,
$$

where "deg" denotes the topological degree. By the homotopy invariance of topological degree,

$$
\operatorname{deg}\left(\phi_{a}(\lambda, \cdot), \Omega(x)\right)=2 \text { for all } \lambda \in(0,1) .
$$

But, for $\lambda$ sufficiently close to $1, \Omega(\lambda)$ is empty and

$$
\operatorname{deg}\left(\phi_{a}(\lambda, \cdot), \Omega(\lambda)\right)=0
$$

This is a contradiction and proves (b).

A sequel [30] to this paper (written after this paper was submitted but accepted before this paper was reported on) extends the method to the Borsuk-Ulam Theorem and analyzes the class of problems for which the homotopy method works.

7. Following the Curve $\Gamma_{a}$. For a number of related problems we have established here that all we need do to find solutions is follow the curve $\Gamma_{a}$. In this section we show that $\Gamma_{a}$ is the solution of a differential equation which is defined on an open set. Hence, the curve may be followed by computer using any of the available differential equation solving algorithms. To be specific, we choose the notation and setting of Theorem 2.4 .

Let $K(\lambda, x)$ be ker $D \phi_{a}(\lambda, x)$; (that is, $D \phi_{a}$ here means $D_{(\lambda, x)} \phi_{a}$ and the ker $D \phi_{a}(\lambda, x)$ is $\left.\left\{v \in R^{n+1}: D \phi_{a}(\lambda, x) v=0\right\}\right)$. Let 0 be the set of $(\lambda, x) \in[0,1) \times$ $B^{n}$ such that the subspace $K(\lambda, x)$ is one dimensional. Let $\left\{e_{1}, \ldots, e_{n}\right\}$ be a complete set of basis vectors for $R^{n}$. Let $(\lambda, x)$ be in 0 . Let $H(\lambda, x)$ be the $n$ dimensional subspace in $R^{n+1}$ which is perpendicular to $K(\lambda, x)$. By construction, for $i=1, \ldots, n$, there exist unique vectors $\tilde{e}_{i} \in H(\lambda, x)$ such that $D \phi_{a}(\lambda, x) \tilde{e_{i}}=e_{i}$. For $v \in R^{n+1}$, let $\mu(v)(=\mu(v, \lambda, x))$ be the determinant of the ordered set $\left(v, \widetilde{e}_{1}, \ldots, \widetilde{e}_{n}\right)$; that is we consider these vectors to be columns of an $(n+1) \times$ $(n+1)$ matrix and evaluate its determinant. Notice that if $v \in K(\lambda, x), v \neq 0$, then $\left\{v, \tilde{e}_{1}, \ldots, \tilde{e}_{n}\right\}$ is a basis for $R^{n+1}$, and so $\mu(v) \neq 0$.

Let $a$ be chosen so 0 is a regular value of $\phi_{a}$. Then 0 is a neighborhood of $\Gamma_{a}$. There are two natural vector fields, that is differential equations on 0 . Write $y$ for $(\lambda, x)$, and let $v^{+}(y)$ be the unit vector in $K(y)$ for which $\mu\left(v^{+}(y)\right)>0$, and let $v^{-}(y)$ be the other unit vector, $-v^{+}(y)$. If $y$ is a point of $\Gamma_{a}$, both $v^{+}(y)$ and $v^{-}(y)$ are tangent to $\Gamma_{a}$ at $y$. Writing $v(y)$ for either $v^{+}$or $v^{-}$, the corresponding differential equation is

$$
\frac{d y}{d s}=v(y), \quad y(0)=(0, a) \in R^{n+1} .
$$


The solution $y(s)$ has $\Gamma_{a}$ as its trajectory and $s$ corresponds to arc length since $|v(y)|=1$. One of these choices traces out $\Gamma_{a}$ with positive $s$, and the other, negative $s$. Let $v_{0}$ be the unit vector in $K(0, a)$ for which the first coordinate, (i.e. the $\lambda$ coordinate) is positive. If $v_{0}=v^{+}(0, a)$, we choose $v(y)=v^{+}(y)$, and $v^{-}(y)$ in the alternative case. Hence, we have now chosen a single differential equation on 0 .

It should be noticed that for any $y \in 0, d \phi_{a}(y(s)) / d s=0$ since $D \phi_{a}(y) v(y)=0$, that is, $\phi_{a}$ is constant along trajectories of (7.1). Of course for an arbitrary point $y \in 0, y \notin \Gamma_{a}$, the solution of (7.1) may trace out a closed loop or it may reach a boundary of $O$ at which $\lambda \neq 1$, that is, a point at which $v$ is not defined.

An alternative approach for defining $v$ (and $v^{+}$and $v^{-}$) is available. Given a point $(\lambda, x) \in[0,1) \times B^{n}$, we could choose $\tilde{a}=\tilde{a}(\lambda, x)$ so that $\phi(\tilde{a}, \lambda, x)=0$. Then if $D_{(\lambda, x)} \phi(\tilde{a}, \lambda, x)$ has rank $n$, we can define $v(\lambda, x)$, namely by choosing it to be tangent to the curve through $(\lambda, x)$ on which $\phi_{\tilde{a}}$ is 0 . Of course, we still have to choose the orientation of $v$ by examining an appropriate determinant. We would expect this second approach to defining $v$ to be better in practice than the first, since in the second we would expect small errors, numerically introduced, to shift the trajectory to a nearby curve which is as good as the original curve. In this alternative approach we would expect the nearby curve by construction to be leading to a fixed point also. Hence, this method may be computationally superior. In fact, there has been insufficient computer experimentation so far with these methods. Certainly pathological examples can be constructed and the frequency of these in practice can only be determined by further experimentation. For discussions of the continuation method in special cases, see also [27]-[29].

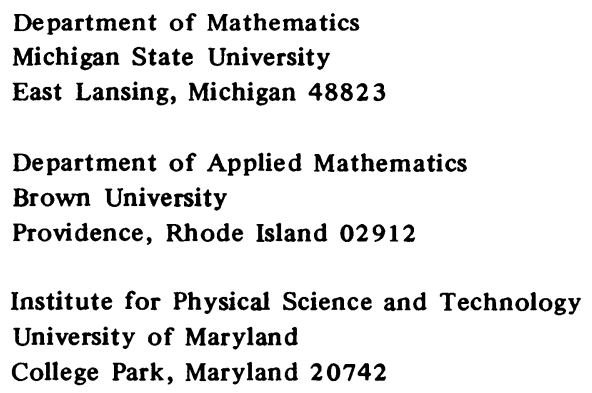

1. H. SCARF, "The approximation of fixed points of a continuous mapping," SIAM $J$. Appl. Math., v. 15, 1967, pp. 1328-1343.

2. B. C. EAVES, “An odd theorem," Proc. Amer. Math. Soc., v. 26, 1970, pp. 509-513.

3. H. W. KUHN, "Simplicial approximation of fixed points," Proc. Nat. Acad. Sci. U.S.A., v. 61,1968, pp. $1238-1242$.

4. B. C. EAVES, "Homotopies for the computation of fixed points," Math. Programming, v. 3, 1972, pp. 1-22.

5. B. C. EAVES \& R. SAIGAL, "Homotopies for the computation of fixed points on un bounded regions," Math. Programming, v. 3, 1972, pp. 225-237.

6. R. T. WILlMUTH, The Computation of Fixed Points, Ph. D. Thesis, Dept. of Operations Research, Stanford University, 1973.

7. R. B. KELLOGG, T. Y. LI \& J. A. YORKE, "A method of continuation for calculating a Brouwer fixed point," Computing Fixed Points with Applications (Proc. Conf., Clemson Univ., 1974), S. Karamadian (editor), Academic Press, New York, 1977, pp. 133-147. 
8. R. B. KELLOGG, T. Y. LI \& J. A. YORKE, "A constructive proof of the Brouwer Fixed Point Theorem and computational results," SIAM J. Numer. Anal., v. 13, 1976, pp. 473-483.

9. S. SMALE, "A convergent process of price adjustment and global Newton methods," J. Math. Econom., v. 3, 1976, pp. 1-14.

10. M. HIRSCH \& S. SMALE, Personal communication.

11. L. S. PONTRJAGIN, "Smooth manifolds and their applications in homotopy theory," Trudy Mat. Inst. Steklov., v. 45, 1955 ; English transl., Amer. Math. Soc. Transl. (2), v. 11, 1959 , pp. $1-114$.

12. M. HIRSCH, "A proof of nonretractability of a cell onto its boundary," Proc. Amer. Math. Soc., v. 14, 1963, pp. 364-365.

13. T. Y. LI, "A rigorous algorithm for fixed point computation." (To appear.)

14. L. WATSON, "Finding fixed points of $C^{2}$ maps by using homotopy methods," Computation and Appl. Math. (To appear.)

15. B. C. EAVES \& H. SCARF, "The solution of systems of piecewise linear equations," Math. of Oper. Res., v. 1, 1976, pp. 1-27.

16. W. C. RHEINBOLDT, "Numerical continuation methods for finite element applications," Formulation and Computational Algorithms in Finite Element Analysis, (Proc. U. S.-German Sympos.), M.I.T. Press, Cambridge, Mass. (To appear.)

17. R. ABRAHAM \& J. ROBBIN, Transversal Mappings and Flows, Benjamin, New York, 1967.

18. J. MILNOR, Topology from the Differentiable Viewpoint, Univ. of Virginia Press, Charlottesville, Va., 1965.

19. A. MAS-COLELL, "A note on a theorem of F. Browder," Math. Programming, v. 6, 1974, pp. 229-233.

20. F. E. BROWDER, “On the continuity of fixed points under deformations of continuous mappings," Summa Brasil. Math., v. 4, 1960, pp. 183-191.

21. J. L. LIONS, Quelques Méthodes de Résolution des Problèmes aux Limites Non Linéaires, Dunod, Paris, 1969.

22. J. C. ALEXANDER, "The additive inverse eigenvalue problem and topological degree," Proc. Amer. Math. Soc., v. 70, 1978, pp. 5-7.

23. S. FRIEDLAND, "Inverse eigenvalue problems," Linear Algebra and Appl., v. 17, 1977, pp. $15-51$.

24. G. SCORZA-DRAGONI, "Sul problema dei valori ai limiti per i system di equazioni differenziali del secondo ordine," Boll. Un. Mat. Ital., v. 14, 1935, pp. 225-230.

25. A. LASOTA \& J. A. YORKE, "Existence of solutions of two-point boundary value problems for non-linear systems," J. Differential Equations, v. 11, 1972, pp. 509-518.

26. M. A. KRASNOSELSKII, Topological Methods in the Theory of Nonlinear Integral Equations, Pergamon Press, New York, 1964.

27. J. M. ORTEGA \& W. C. RHEINBOLDT, Iterative Solution of Nonlinear Equations in Several Variables, Academic Press, New York, 1970.

28. J. DAVIS, The Solution of Nonlinear Operator Equations with Critical Points, Ph. D. thesis, Oregon State Univ., 1966.

29. G. MEYE R, "On solving nonlinear equations with a one-parameter operator imbedding," SIAM J. Numer. Anal., v. 5, 1968, pp. 739-752.

30. J. ALEXANDER \& J. A. YORKE, "The homotopy continuation method: Numerically implementable topological procedures," Trans. Amer. Math. Soc., v. 242, 1978, pp. 271-284. 